\title{
Parallelizing Training of Deep Generative Models on Massive Scientific Datasets
}

\author{
Sam Ade Jacobs Brian Van Essen David Hysom Jae-Seung Yeom Tim Moon Rushil Anirudh \\ Jayaraman J. Thiagaranjan Shusen Liu Peer-Timo Bremer Jim Gaffney Tom Benson Peter Robinson \\ Luc Peterson Brian Spears \\ Lawrence Livermore National Laboratory
}

\begin{abstract}
Training deep neural networks on large scientific data is a challenging task that requires enormous compute power, especially if no pre-trained models exist to initialize the process. We present a novel tournament method to train traditional as well as generative adversarial networks built on LBANN, a scalable deep learning framework optimized for HPC systems. LBANN combines multiple levels of parallelism and exploits some of the worlds largest supercomputers.

We demonstrate our framework by creating a complex predictive model based on multi-variate data from high-energydensity physics containing hundreds of millions of images and hundreds of millions of scalar values derived from tens of millions of simulations of inertial confinement fusion. Our approach combines an HPC workflow and extends LBANN with optimized data ingestion and the new tournament-style training algorithm to produce a scalable neural network architecture using a CORAL-class supercomputer. Experimental results show that 64 trainers (1024 GPUs) achieve a speedup of $70.2 \times$ over a single trainer (16 GPUs) baseline, and an effective $109 \%$ parallel efficiency.
\end{abstract}

Index Terms-machine learning, large-scale, generative models, parallel computing

\section{INTRODUCTION}

The explosion of deep learning in recent years has unlocked the potential to change the way in which we tackle large scale scientific simulations. While machine learning techniques are being applied to many academic, medical, and commercial applications, the field of large scale, scientific machine learning techniques is just beginning to emerge. The scientific community is leveraging advances in deep learning and computational workflows to bridge the gap between approximate scientific simulations and more accurate but expensive and often limited experimental analysis. We refer to this coupling of traditional scientific computing with deep learning as cognitive simulation and envision it as a new way to enable predictive science. There are many challenges to this new methodology including generating large scale scientific data sets for training, developing learning methods for unlabeled data, scaling up deep learning training methods to leverage state of the art supercomputers, and developing novel neural network architectures for scientific applications. In this paper we present our approaches that address these

(C2019 IEEE challenges, with a particular focus on the development of parallel methods for large scale training of generative models.

The overarching goal of our cognitive simulation research is the development of techniques that can use machine learning to augment workflows and to supplement, combine, or replace existing heuristics. Depending on where these models engage the simulation they are referred to as being 1) in-the-loop, 2) on-the-loop, or 3) around-the-loop [1]. Notionally, examples of these levels of engagement are 1) directly in a physics simulation, 2) observing and influencing a simulation code, or 3) part of the simulation campaign. Using machine learning (or specifically deep learning) at each of these levels has different requirements for the size of admissible models and the required speed of inference. In this paper we present our work on developing novel generative models that take physical constraints into account and are used around-theloop of an inertial confinement fusion (ICF) simulation campaign. Here we use results from a recently proposed semianalytic simulation model [2], [3]. to simulate the behavior of the implosion of a fuel capsule in instruments such as the National Ignition Facility (NIF) at Lawrence Livermore National Lab (LLNL). These types of simulations are critical to understanding the physics occurring during an implosion and subsequently improving the output of the experimental campaigns.

Scientific machine learning presents the researcher with several unique challenges. First and foremost, scientific data sets that come from large-scale simulations or experiments can be extremely large, are frequently high dimensional, and require domain expertise to label the data. Secondly, because we are applying novel analysis to uncommon data there is a lack of predefined neural network models or proven model families to draw upon when starting a new analysis. As such, new neural network architectures have to be designed and tuned for specific problems and data sets. This requires massive training of hyperparameters and model exploration.

In this paper, we present our work on extending a scalable deep learning framework with a novel tournament parallel algorithm that is able to train large, complex generative models on massive amounts of multimodal data. Additionally, we have developed a novel, distributed, in-memory data store that is optimized for minimizing file system access during training of deep neural networks. We integrated these 
algorithms and capabilities into the Livermore Big Artificial Neural Network (LBANN) deep learning framework. LBANN is an open source HPC-centric framework built on the Hydrogen distributed linear algebra and Aluminum GPUaware communication libraries [4]-[8]. The novel tournament algorithm, Let a Thousand Flowers Bloom (LTFB), primarily targets scaling up the training of deep neural networks on massive data sets and leveraging leadership-class HPC systems. The main thrust of LTFB is to minimize the amount of synchronization required for each step of the training algorithm and to develop a mechanism for combining independently trained models. The key feature of LTFB is the independent training of models on partitioned, more manageable data sets, while yielding a model that is as good a one trained on the entire data set. This allows for strong scaling and is accomplished by periodically running a tournament where a locally trained model competes with other models on a held-out "tournament" data set. The winning model continues and losing models are discarded. Propagation of the winning model serves as an efficient encoding of key features from other parititons of the data. The LTFB algorithm provides a mechanism for scalable data ingestion by allowing partitioning of the data set without loss of generalizability. Furthermore, it enables scalable exploration of the initial state space as well as the state space after each tournament. Implementing LTFB within the LBANN framework and coupling it with the datastore allowed us to efficiently train on a $2 \mathrm{~TB}$ database of 10 million 5-D input parameters, 120 million multispectral images and 10 million 15-D scalar values. This is the first demonstration of learning at such scale on a multi-variate scientific data set.

In addition to our work on large scale learning, developing a cognitive simulation capability requires innovation in scientific workflows and neural network architectures. We briefly present an overview of these capabilities in Sections III The LTFB algorithm and data-store are discussed in Section IIII followed by our experiments in Section IV, and related work in Section $\mathrm{V}$. In summary, we show that LTFB provides the ability to strongly scale the training time of a single neural network architecture, taking advantage of thousands of GPUs, while maintaining a reasonable sized mini-batch. This capability provides a unique ability to explore complex neural network architectures, while using unsupervised learning methods on massive data sets. We summarize our contributions in this paper as follows:

- A scalable deep learning framework that is able to strongly scale training of a single model on thousands of GPUs, while only requiring modest data parallelism.

- A novel tournament method, LTFB, that is optimized for complex generative models, minimizes communication, and enables efficient partitioning of large data sets while maintaining model generalizability.

- A new in-memory distributed data-store optimized for training deep neural networks, which leverages low-latency, high-bandwidth interconnect for efficient file system access.

- First demonstration of scalable training of complex cyclic

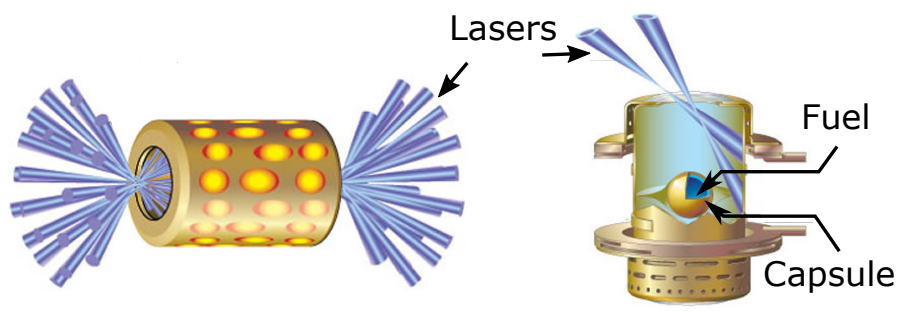

Fig. 1. Schematic of an ICF experiment at NIF. High energy lasers heat and compress a target capsule containing thermonuclear fuel, resulting in a fusion process.

generative models to 1024 GPUs without loss in quality.

\section{SCIENTIFIC Problem}

\section{A. Objective}

This work was developed to support inertial confinement fusion (ICF) experiments at the National Ignition Facility (NIF). These experiments use the world's largest laser to heat and compress a millimeter-scale target filled with frozen thermonuclear fusion fuel (see Figure 11. Under sufficiently well-controlled conditions, the compressed fusion fuel will produce enough energy for the target to self-heat, leading to a runaway implosion process called ignition. Ultimately, ignition will produce more fusion energy than the driving laser energy [9], enabling study of fusion energy production, astrophysical phenomena, and nuclear weapons processes.

Our particular goal was to develop a fast surrogate model that can predict the outcome of ICF experiments. It could be used, for instance, for experiment optimization, statistical uncertainty quantification, or efficient sampling of the experimental parameter space. Robust model inversion could also be used to infer the physics processes underlying experimental observations. Each ICF experiment generates a rich signature set composed of scalars and images, so the surrogate model would be a large generative model for complicated multimodal data. Due to the complexity and sensitivity of ICF experiments, a large volume of simulated data was necessary to train such a model.

\section{B. JAG simulator for ICF}

First-principles simulation of ICF implosions require highfidelity multiphysics simulations [10], frequently taking thousands of CPU-hours per sample. To produce the required volume of data, we instead used the JAG model, a semianalytical model for the final stages of an ICF implosion [2]. Since all experimental signals are generated during this final period, JAG is capable of generating a realistic set of multi-modal outputs while only taking a few CPU-seconds. We performed simulations over a 5-dimensional parameter space - controlling the strength of the laser drive and the 3D shape of the imploding shell - and simulated X-ray cameras on three different lines of sight - each with 4channel hyperspectral energy resolution and spatial resolution of $64 \times 64$ pixels. We also postprocessed the JAG output to 
obtain 15 scalar-valued observable signatures. Thus, each data sample is a pair consisting of an input 5-vector and an output bundle of 15 scalars and 12 images. Generally, varying the drive parameters resulted in highly non-linear variations in the scalar performance metrics and varying the shape parameters resulted in major changes in the X-ray images.

\section{Ensemble workflow}

Given the strong non-linearities in ICF experiments, it was important to densely cover the five-dimensional parameter space for JAG simulations. This was challenging not only because of the large number of runs and corresponding files, but also because JAG is so fast. Running JAG and performing postprocessing only takes about a minute, so a workflow system's runtime can be dominated by the overhead of scheduling, placing, and executing jobs. We addressed this problem with an extension of the Merlin workflow system [11], which uses a custom combination of various open-source components to build a highly flexible and efficient framework. We used a spectral sampling approach to optimally assign simulation parameters [12], resulting in 10 million simulations for the training dataset and 1 million for the test dataset. To manage this enormous number of samples, we packaged the data into 10,000 HDF5 files, each of which contains 1,000 samples.

\section{Neural network architecture}

The surrogate model was implemented as the CycleGAN shown in Figure 2 since it imposes several desirable consistency conditions. First, internal consistency means that the forward model predicts all of the output modalities jointly. This avoids the uncorrelated errors and physically invalid solutions that can arise if each output modality is predicted independently. Second, physical consistency means that predictions are realistic, ideally to the point where they are statistically identical to the training data. We approximate this by training an adversarial discriminator model to distinguish predictions from data samples [13]. This is technically not a physical constraint, but it does markedly improve the quality of predicted images. Third, self consistency means that there is an inverse model that projects back to the original input. This is particularly useful since both the forward and inverse models would be useful for domain scientists in ICF. From a machine learning perspective, consistency between the forward and inverse models also acts as regularization on an otherwise highly underdetermined problem.

More precisely, the forward model $\mathcal{F}: \mathbb{R}^{5} \rightarrow \mathbb{R}^{20}$ maps from the 5-D experiment parameter space to a 20-D latent space. This is trained a priori using a multimodal autoencoder of all outputs. Predicted scalar values and X-ray images are obtained by passing latent space vectors into a decoder network and the internal consistency condition is enforced with the mean absolute error loss. The discriminator model $\mathcal{D}: \mathbb{R}^{20} \rightarrow\{0,1\}$ is trained adversarially. The inverse model $\mathcal{G}: \mathbb{R}^{20} \rightarrow \mathbb{R}^{5}$ attempts to enforce the self consistency condition $\mathcal{G} \circ \mathcal{F} \approx \mathcal{I}$ using the mean absolute error loss.

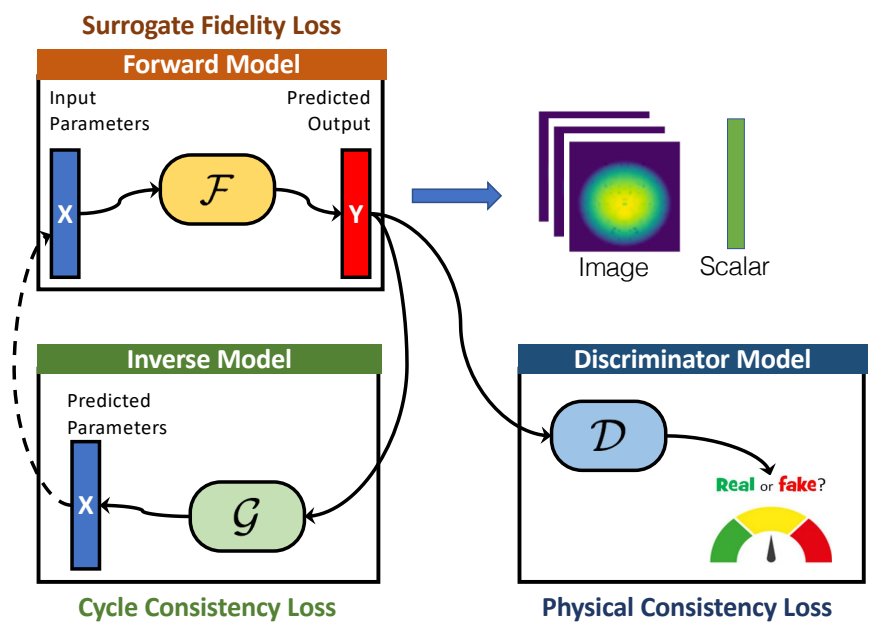

Fig. 2. CycleGAN surrogate model for ICF experiments.

Each of these components is implemented as a standard fullyconnected neural network. A complete description of the network is available at [14].

\section{Scalable DeEP Learning}

We used LBANN [4], [5], an open-source deep learning framework from LLNL, as the platform for this research. LBANN implements a suite of algorithms for training deep learning models on distributed memory architectures, exploiting both data- and model-parallelism to achieve scalable performance on HPC systems. We have extended LBANN in this work to support GANs and have also upgraded the internal infrastructure to improve robustness and scalability when training multiple concurrent models. We have developed a distributed in-memory data store that minimizes access to the parallel file system during training. Finally, we have extended the LTFB algorithm presented by Jacobs et al. [15] to handle GANs.

LBANN's software stack is shown in Figure 3 The toplevel framework is written in C++ and CUDA. Hydrogen [6], a fork of Elemental [16], provides distributed linear algebra with GPU acceleration and Aluminum provides GPU-aware asynchronous communication [8].

\section{A. Trainers and models}

Two key concepts in LBANN are those of trainers and models. A trainer is a collection of compute resources that operate together as a unit. A model is a neural network, comprised of a directed acyclic graph (DAG) of tensor operations ("layers"), trainable parameter tensors ("weights"), and data readers. Naturally, trainers are responsible for training models, usually with a variant of stochastic gradient descent. During a model's mini-batch step, the trainer will ingest and preprocess data with a data reader and pass it into the model's DAG. Each trainer manages one or more models and it may accelerate computation with data-parallelism, modelparallelism, or both. Observe that running LBANN with 


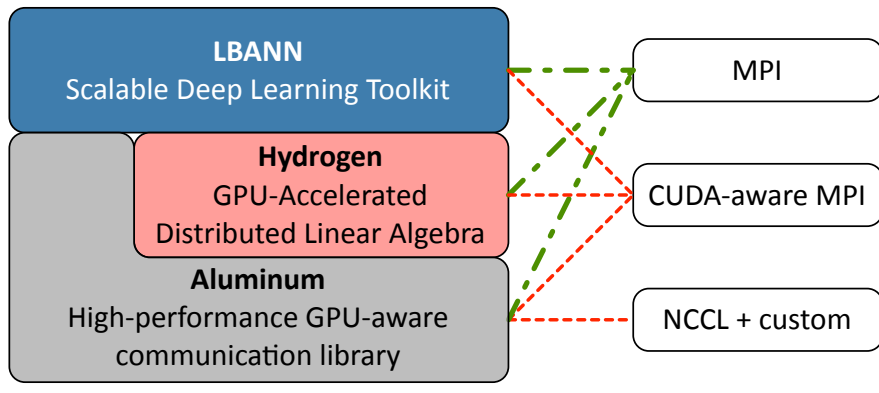

-- CPU-Only ---- GPU-Accel

Fig. 3. LBANN's software stack, consisting of the Hydrogen linear algebra library and the Aluminum communication library.

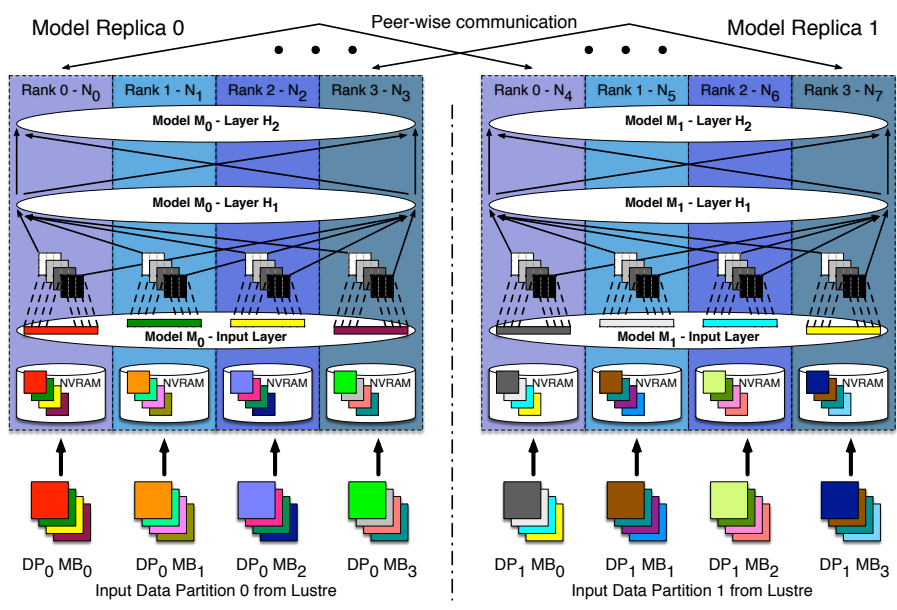

Fig. 4. Example of LBANN running with two trainers, each of which consists of four MPI ranks.

multiple trainers results in two levels of parallelism: within each trainer and between trainers.

\section{B. Data store}

One of the key challenges of this work was training data management. Typically, data from large simulation runs are generated, triaged, and dumped to a parallel file system like Lustre or GPFS. A naive data reader would then populate each mini-batch by opening the files for its required data samples. However, recall from Section [I] that each data file consists of 1,000 samples. In addition, samples should be drawn randomly from the dataset to make sure they are representative of the source distribution. Consequently, each process will open many files and each file may be accessed by multiple processes at the same time. This puts a huge burden on the file system and drastically slows down data ingestion. In a naive approach to $\mathrm{I} / \mathrm{O}$, data ingestion can dominate the training time of the model.

To avoid this problem, we have implemented a scalable, distributed in-memory data store in LBANN. Each process in a trainer is assigned to manage a subset of the data to be cached in system memory. Therefore the total data store capacity is proportional to the number of compute nodes in the trainer.

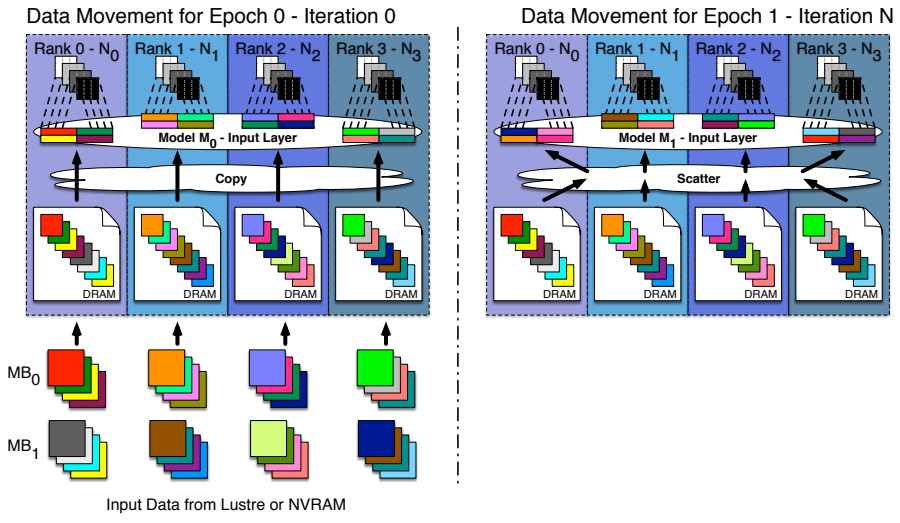

Fig. 5. Example of an LBANN data store for a trainer with four MPI ranks. The data store is populated dynamically during the first training epoch, after which no data is read from the file system.

At each training step, processes will distribute the locallycached samples that they manage to the trainer's MPI rank that requires them for the upcoming mini-batch. This shuffling is done with non-blocking communication on background threads, so it efficiently overlaps with other computation. The data store itself utilizes Conduit to provide a data-typeagnostic in-memory framework for managing data samples [17]. While some popular open-source frameworks provide local data caching on a single node [18], [19], this work presents - to the best of our knowledge - the first distributed in-memory data store in a deep learning framework.

We have explored two approaches to populate this data store, dynamic and preloading. In the dynamic approach, data samples are read from data files during the first training epoch in a similar manner as naive data ingestion, but samples are cached in the data store as they are used. Thus, we only expect to suffer the previously discussed performance penalties during the first epoch, after which no data is read from the file system. For each subsquent epoch, data is incrementally shuffled between the ranks at each mini-batch step. Preloading is a second optimization technique that fully populates the data store prior to training. While this shifts the file I/O burden to a preprocessing step, it allows for optimal access to samples from multi-sample file formats such as HDF5. To preload the data store, each process is assigned a disjoint subset of the data files and accesses, in parallel, all of the data samples within each file. This minimizes the number of files each process opens concurrently, and ensures that each file is only opened by one process per trainer. During training itself, no data is read from the file system.

\section{LTFB for GANs}

"Let a Thousand Flowers Bloom" (LTFB) is a decentralized variant of population-based training [15] [20], primarily intended to achieve scalable performance when training neural networks with massive datasets on HPC systems. It begins by initializing multiple trainers and partitioning the training dataset between them. The trainers construct models and train them in a loosely-coupled manner. Training each model 


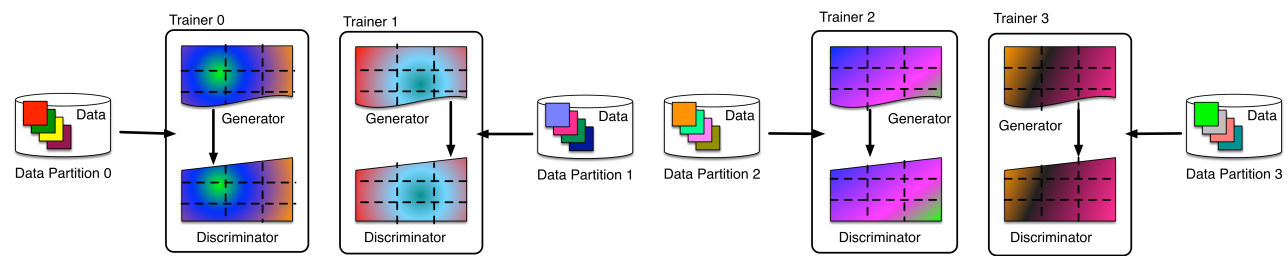

(a) Trainers independently train GANs using a partition of the training dataset.

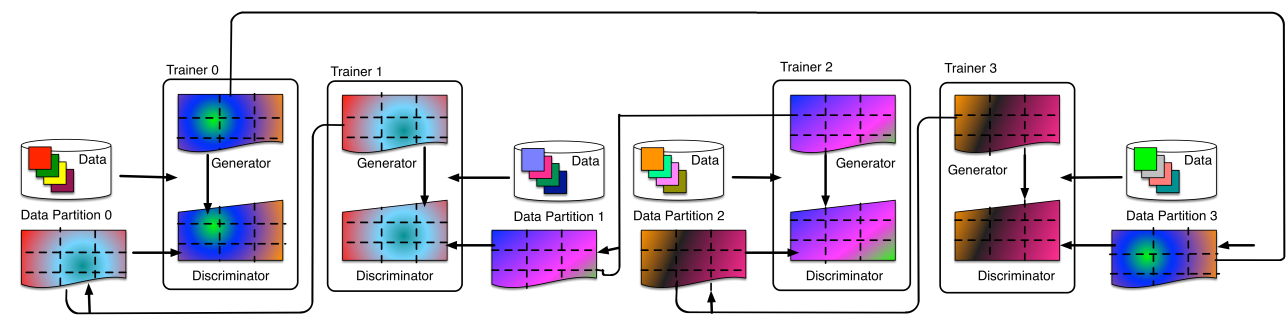

(b) During an LTFB round, trainers will pair up, exchange their generators, and evaluate them against their local discriminators. The better generator is retained for further training.

Fig. 6. Example of training GANs with LTFB using four trainers. Dashed lines in each trainer represent that the models (or data) are internally parallelized across GPUs.

between tournaments is independent, and the tournament provides periodic coupling between the trainers. The models are initialized with different weights and hyperparameters, but even identical models will diverge over the course of training since trainers expose different silos of data. Periodically, e.g. at predefined mini-batch intervals, trainers are randomly paired up and made to exchange models. Each trainer will evaluate its two models on a local tournament data set, keeps the one that achieves a better evaluation metric, and then resumes training. Even though each trainer only exposes a model to a subset of the data, models that survive LTFB are likely have been exposed to many trainers at different times, and thus are expected to capture the characteristics of the entire dataset.

This approach has some nice scaling properties. Communication between trainers is limited to infrequent peer-to-peer model exchanges, so increasing the number of trainers does not incur significant performance overheads. Furthermore, if a trainer is efficiently mapped to the hardware topology, e.g. to a compute node, intra-trainer communication is well optimized and the number of processes is typically small enough to fruitfully apply strong-scaling techniques. Finally, the fact that the dataset is divided amongst the trainers helps keep file I/O scalable.

Previous work on LTFB demonstrated modest scaling on image classification benchmarks [15]. We extended that work to GANs by only exchanging generator models during LTFB rounds and keeping discriminators local to each trainer, as shown in Figure 6. This approach intuitively mimicks the practice of educating a student with multiple teachers, which has been shown to improve the quality of trained generative models on the MNIST, CIFAR-10 and CelebA benchmarks [21]. It also reduces the inter-trainer communication volume during each LTFB round.
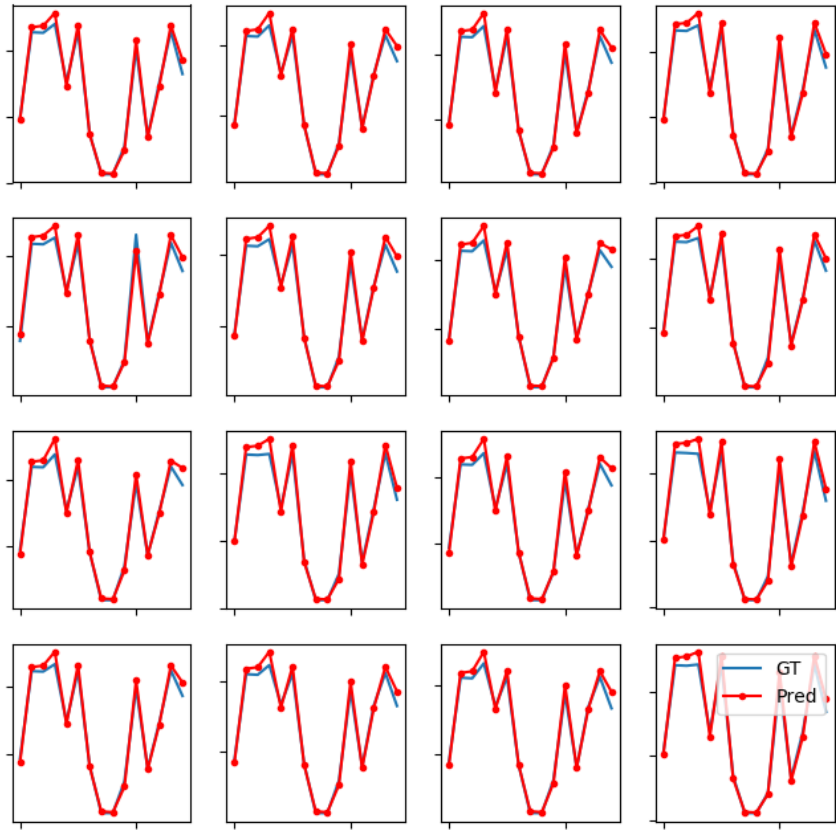

Fig. 7. Ground truth and LTFB CycleGAN predicted 15-D scalars for 16 validation samples. Note that the ground truth in blue is mostly covered by the GAN's prediction in red.

\section{EXPERIMENTS}

The LTFB algorithm enables a new dimension for strong scaling deep learning training. We achieve strong scaling by allowing the global data set to be split into smaller partitions and still yielding a trained model that generalizes well on the entire testing data set. The critical contribution of the LTFB training method is that the winning model has learned enough from each trainer and associated data partition to accurately capture the characteristics of the entire training data 


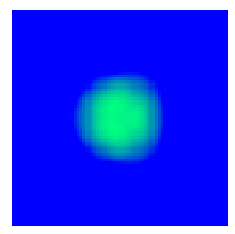

(a) Ground truth at view0 channel0

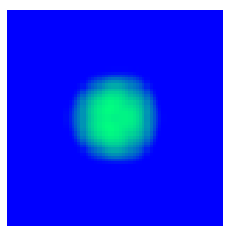

(b) Predicted at view0 channel0

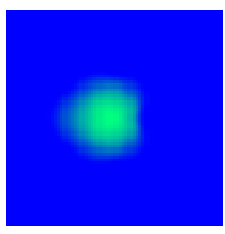

(c) Ground truth at view1 channel1

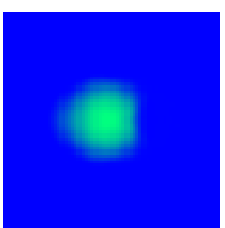

(d) Predicted at view1 channel1
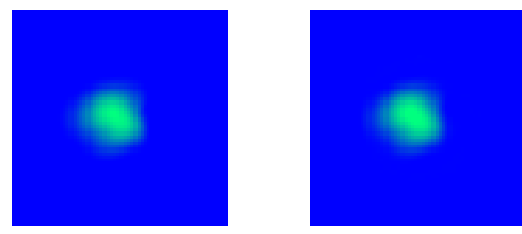

(e) Ground truth at view2 channel2 (f) Predicted at view2 channel2

Fig. 8. Capsule images (at selected views and channels) from the JAG model's output (ground truth) and generated by the LTFB CycleGAN generator network.

set. Essentially, when a model is shared it is acting as an encoded representation of its data partition. Thus transfering that learned representation to the new trainer for continuing education. As we will demonstrate in these experiments, the ability for LTFB to strong scale with a given mini-batch size allows it to unlock a new axis of parallelism that is independent of, and composable with existing data and model parallel methods.

To demonstrate the efficacy of the LTFB algorithm we run multiple experiments that show the limits of strong scaling via data parallelism, the challenges of balancing data ingestion and parallel training, a comparison of a plain ensemble method, and the ability of LTFB to provide strong scaling while producing a model of equivalent or better performance. It is important to note that for these experiments we worked with a consistent set of model architecture and hyperparameters for the CycleGAN network described in Section II] Over the course of developing the network and curating the data set, we identified that a mini-batch size of 128 samples, an adam optimizer, and an initial learning rate of 0.001 work well for these experiments. Figures 7 and 8 show some ground truth scalars and images respectively alongside predictions from our model.

\section{A. Setup}

Our experiments are run on the Lassen supercomputer at Lawrence Livermore National Laboratory, within the Livermore Computing collaboration zone (LC CZ). Lassen is a CORAL-class system (like Sierra), with 795 nodes, each of which consists of two IBM POWER9 CPUs and four Nvidia Volta V100 GPUs. The GPUs and CPUs are interconnected with three NVLINK2 connections and each Volta has $16 \mathrm{~GB}$ of memory the node has $256 \mathrm{~GB}$ of system memory. Nodes are interconnected via dual-rail InfiniBand EDR. Our implementation of LTFB and the data store were build on a recent development version of LBANN, Hydrogen, and Aluminum. Our software development environment used GCC 7.3.1, Spectrum MPI 2019.01.30, CUDA 9.2.148, cuDNN 7.5.0, and NCCL 2.4.2. For all results we use singleprecision floating point data types.

\section{B. Small-scale data parallelism}

The most common method of strong scaling model training time is data parallelism: where the samples within a mini-batch

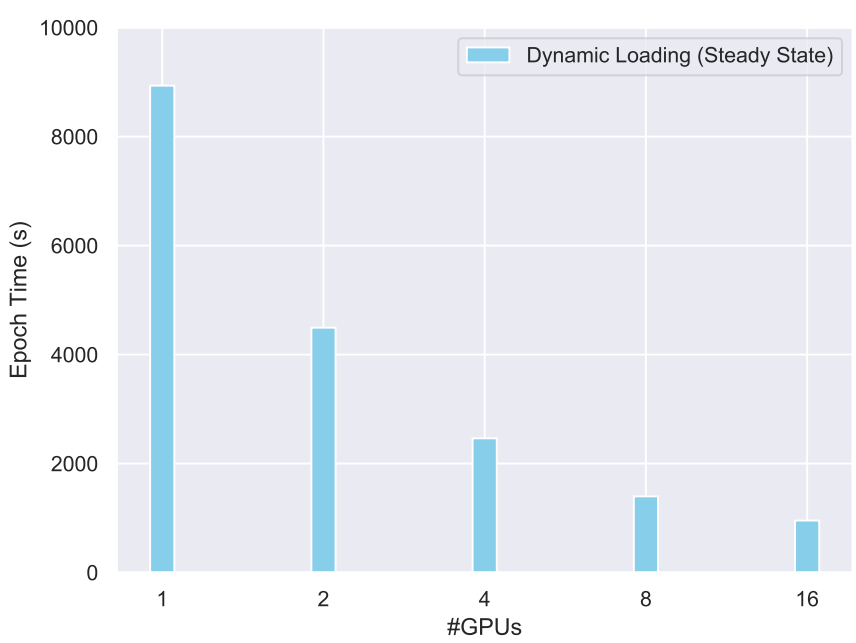

Fig. 9. Evaluating the benefit of increasing the number of GPUs used for data parallel training a single model.

are distributed across multiple nodes and partial error signals and gradient updates are aggregated via all-reduce collectives during back propagation. The ability of this method to scale is limited by the global mini-batch size and the amount of compute required per node as it will become communication bound. Figure 9 shows the results of training the CycleGAN on using only $1 M$ samples from the full $10 M$ sample data set. With simple data parallel scaling from 1 node and only 1 GPU training the CycleGAN to using 4 nodes and 16 GPUs there is a $9.36 \times$ improvement in steady state epoch time. Figure 9 does show that the benefits of data parallel scaling are starting to diminish around 4 nodes and 16 GPUs, with a decrease in parallel efficiency down to $58 \%$. To provide a good balance between data parallel and independent trainers for the tournament experiments, we use 4 nodes and 16 GPUs to train each model in subsequent sections.

\section{Exploring the Data-store}

One of the challenges of working with large scale scientific data sets is that the cost to ingest the training, evaluation, and testing data can easily become a bottleneck or complete impediment to training the network. As noted in Section II] the ICF data set is stored in 10,000 HDF5 files with 1,000 samples per file. When the data was generated the samples were stored 


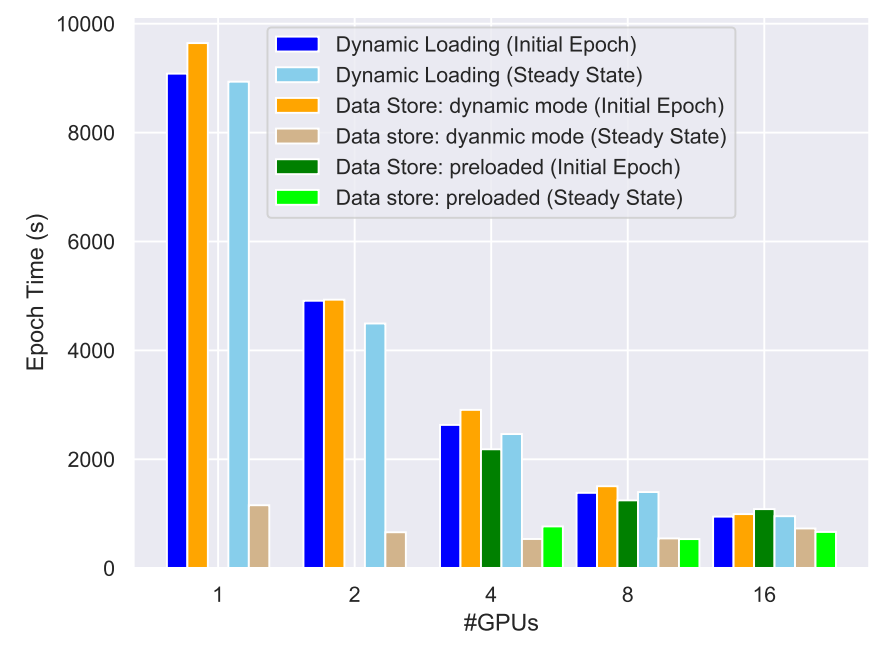

Fig. 10. Evaluating training the CycleGAN model with the data store in dynamic mode, preloaded and without the data store on $1 M$ sample data set.

in these HDF5 bundles in the order in which the 5-D input space was explored by the semi-analytical simulation tool. Therefore, to effectively train the neural network it is necessary to randomly sample from all of the available files to pull a uniform distribution for each mini-batch and thus each step of the Stochastic Gradient Descent (SGD) algorithm. This type of access pattern creates a significant burden on the parallel file system and leads to training runs that are dominated by the overhead of data ingest. 1

As described in Section III-B, we have developed an inmemory data store that caches the training, evaluation, and potentially test data sets in the system's host memory. Figure 10 show the result of increasing the data parallelism by varying the number of nodes used by the trainer using a $1 M$ sample data set on the Lassen system. On the $\mathrm{x}$-axis is the number of GPUs used to train the model and the y-axis is the epoch time in seconds. The results are presented for three configurations, each of which shows the results for the inital and steady state epochs. In Figure 10 for each number of GPUs on the xaxis, the left three bars show the initial time to load the data set and complete the first epoch for each configuration. The right three bars are the cost of each subsequent epoch as the system enters steady state. Note that the configurations with the preloaded data store did not have sufficient memory to load the model with 1 or 2 GPUs. We see from Figure 10 that using the data store has substantial benefit ranging from a massive $7.73 \times$ for a trainer using a single GPU to a $1.31 \times$ for a trainer with 4 nodes. When training with a fairly small data set like the $1 \mathrm{M}$ subset for this experiment, preloading the data has limited advantage over the dynamically loaded data store. Figure 10 shows that for 4 nodes there is a $1.43 \times$ improvement

\footnotetext{
${ }^{1}$ Note that for the purposes of this test we could have shuffled the data and repacked the HDF5 files to provide a random distribution per file, but that optimization is infeasible in real scientific workflows. Additionally, it would not solve the problem of requiring a unique distribution for each subsequent epoch.
}

versus no data store, and a $1.10 \times$ improvement over the dynamically loaded data store. However, the advantage of preloading substantially improved when transitioning to the $10 M$ sample data set due to the larger number of random file access required by the dynamically loaded data store. Therefore for the next experiment we focus on using 4 nodes per trainer and preloaded data store.

\section{LTFB at scale}

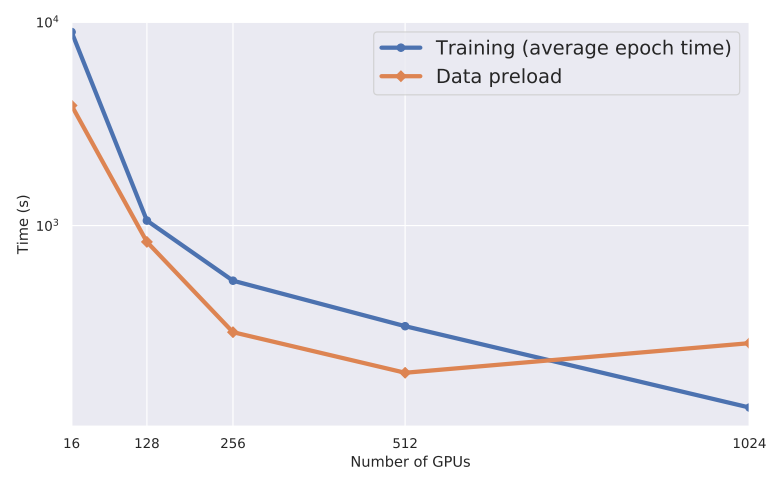

Fig. 11. LTFB training times for CycleGAN network on $10 M$ sample data set

The previous two experiments demonstrate that data parallelism within a single trainer can significantly improve the time required for data ingestion, but performance will quickly plateau when using a fixed size mini-batch. ${ }^{2}$ This experiment shows that LTFB is able to strong scale the training time of a model without a loss of generalization. We use a $10 M$ sample sub-set of the entire $11 M$ sample data set (leaving $1 M$ for validation) that is described in Section III, and scale up to 1024 Volta GPUs on 256 nodes of the Lassen system.

In Figure 11 we show the per-epoch training time in steadystate as we increase the number of LTFB trainers from 1 up to 64. The $\mathrm{x}$-axis is the number of GPUs used across all trainers, with each trainer using 16 GPUs across 4 nodes. This corresponds to $1,8,16,32$, and 64 trainers in the experiment of Figure 11 Note that with 4 nodes per trainer, the data store of a single trainer was unable to load the entire $10 M$ sample training data set due to memory capacity. Therefore for the single trainer case we used 16 nodes per trainer and only 1 GPU per node. This increased memory capacity allowed the data store to load both the training and validation data. As noted previously, for the rest of the configurations, we used 4 nodes and 16 GPUs per trainer. As the number of trainers increase, the steady-state epoch time decreases because each trainer is responsible for a smaller partition of the data set. Figure 11 shows that 64 trainers achieve a speedup of $70.2 \times$ over the 1 trainer baseline, and an effective $109 \%$ parallel

\footnotetext{
${ }^{2}$ As noted previously, large-scale mini-batch training is a regime that is compatible with the LTFB algorithm but does require significant tuning of the learning rate and has not been shown to generalize well to all problem domains.
} 


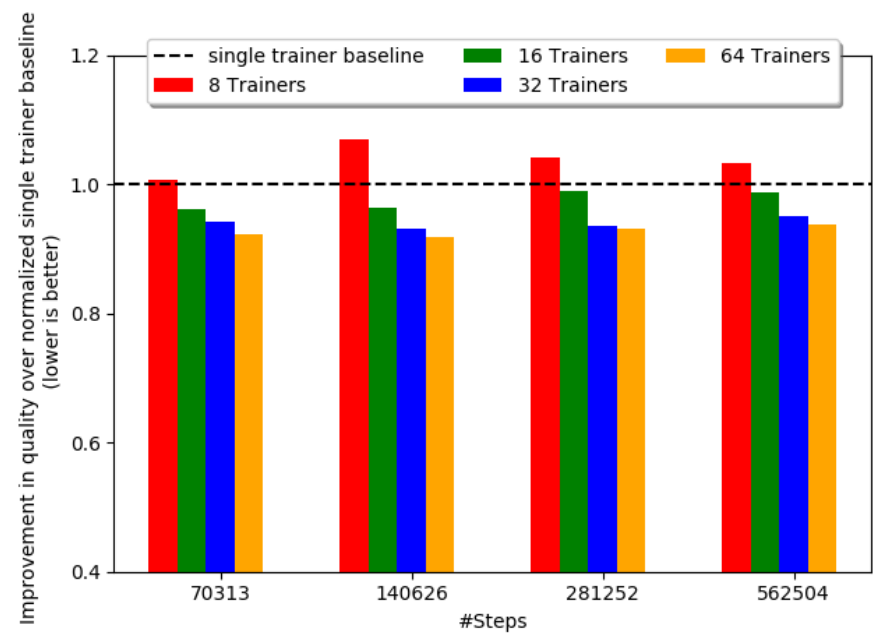

Fig. 12. Improvement in quality (validation loss) over single-trainer baseline at different iterations (steps) per-trainer count.

efficiency with no loss in model quality. Such superlinear speedups in strong scaling are due to "cache" effects as the aggregate working set size is increased and demonstrates non-linear performance improvements. Looking at the time required for preloading the data in Figure 11 we see that at 64 trainers, the total time for all trainers to load the data has degraded over the 32 trainer test point. This loss in performance is due to contention at the GFPS parallel file system resulting from inter-trainer interference and will be addressed in future work.

As noted earlier, the key attribute of the LTFB training algorithm is that the quality of these models actually improves with the number of trainers. While each trainer is responsible for a smaller share of the global data set, the tournament and model exchange in LTFB produces a model that has been trained on a sufficient subset of the data to provide good generalizability (as measured by forward and inverse loss on global validation data set). Figure 12 shows improvement in quality normalized over single-trainer baseline at the different per-trainer iteration. Figure 12 in combination with Figures 11 show that LTFB does not suffer quality degradation with improved parallel performance. LTFB at bigger trainer sizes shows improved learning quality and time to solution if measured by per-trainer number of iterations (steps) which roughly translates to wall clock time.

Figures 8 and 7 show ground truth and predicted images and 15-D scalars of selected samples from the 1 million validation dataset. These two figures show the quality of a trained multimodal surrogate model that jointly predict realistic images and scalars. The plots show predicted images from different viewpoints and scalar values that closely correlate with ground truth values.

\section{E. Comparing LTFB vs partitioned K-independent training}

One simpler alternative to LTFB would be to train Kindependent models and simply select the best final result.
However, that assumes that a single trainer can hold the entire dataset during training which is typically not the case. For example, we were not able to process the data with only four trainers (using 4 nodes per trainer) as a quarter of the dataset proved too large. As the number of trainers used increases, each trainer see fewer samples thus making the data set size more manageable - identical to the behavior of the LBANN trainers during the LTFB. However, while the LTFB model exchange allows each trainer to create models that have learned on compositions of the entire data set, every trainer in the $\mathrm{K}$-independent algorithm is restricted to an ever diminising portion of the full data set. As a result, the models created by the K-independent algorithm generalize more poorly on a held out validation set.

To demonstrate this trade-off, Figure 13 shows comparisons between running LTFB with $k$ trainers vs. $k$ independent trainers using a random $1 / k$ subset of the data. This compares roughly equal runtimes (i.e. equal number of iterations) and equal memory footprints between the two approaches. As shown in the figure, the LTFB approach consistently achieves better results in validation loss. More importantly, with increasing $k$ the gap widens, which is unsurprising as the independent models see progressively smaller portions of the training data and thus generalize less well. This means that for small data in which we can hold all or a significant portion of the training data in memory the $\mathrm{K}$-independent training approach is viable. However, as the size of data sets increases the K-independent training approach breaks down. Another viewpoint to consider is the likelihood of any one independent trainer selecting a small subset of the training data that is distributed sufficiently well to lead to a generalizable model. As the data becomes larger, our chances for this oracular selection will drastically decrease and in the limit they may not even exist. Using the LTFB training algorithm instead produces models with better results for the same compute resources and the datasize is only limited by the memory capacity of the entire system rather than that of a single trainer.

\section{RELATED WORK}

Recent successes of deep learning in many application domains have encouraged renewed interest in understanding and optimizing neural networks. Of particular interest and related to our work is the subject of scaling neural network training on different architectural platforms. Distributed and parallel training of deep learning algorithms is well-studied but mostly focused on the traditional fields of computer vision and speech recognition [22], [23], [24], [25]. [26], [27], [28], [29]-[31]. Data and model parallelism in these work are orthogonal to our work and are used within each trainer to maximize parallelism. Here, we leverage ideas from these existing domains but focus on generative surrogate model for fusion science application and target HPC systems.

The LTFB algorithm was initially proposed in [15] and demonstrates modest scaling on image classification benchmarks. At about the same time, researchers at Google Deepmind explored a similar idea for hyperparameter 

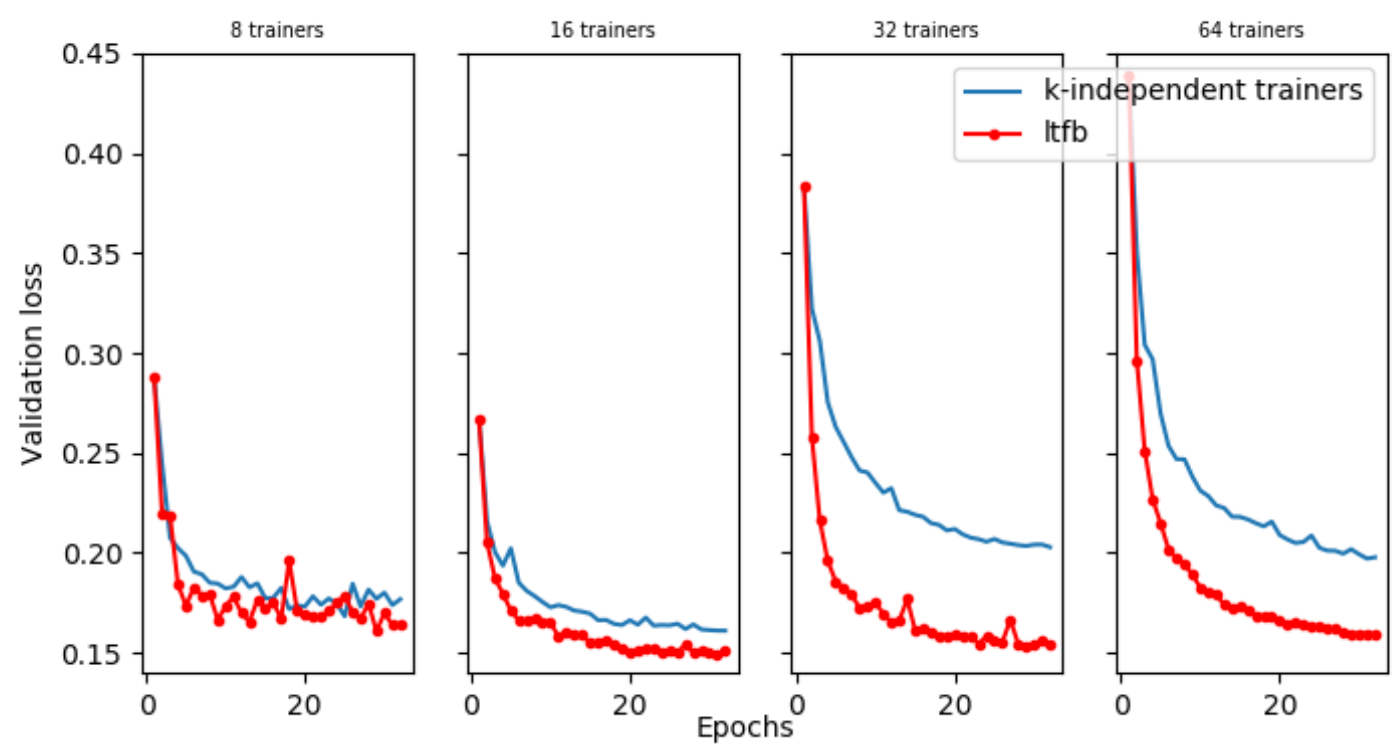

Fig. 13. Validation loss comparison of LTFB vs partitioned K-independent training for CycleGAN (lower is better)

exploration without data partitioning [20]. Deepmind's work did not address scaling but demonstrate the utility of population-based training approaches for hyperparameter optimization on a number of computer vision and reinforcement learning benchmarks. In this work we present new methods for applying these tournmanet methods to generative adversarial networks, extend them to a different (scientific) domain with multivariate data, and provide a primary focus on large scale training using HPC systems.

Scientific machine learning is drawing attention to problems that are beyond computer vision and natural language processing [1], [32]. Prominent recent works are: exascale deep learning for climate analytics [33], and CosmoFlow that used deep learning to learn the universe at scale [34]. In both cases, straight forward large-batch data parallelism is used without addressing convergence issues or the quality of trained regression network

The climate analytics and cosmology problems mentioned above emphasize the challenges of data ingestion and present novel opportunities to improve performance. Kurth et al. [33] developed distributed data staging system in which each rank first copies a disjoint subset of the entire dataset from the parallel file system into its fast local storage, and then distributes the files to other ranks that require them using the point-to-point MPI communication. This significantly reduces the I/O bottleneck at the filesystem. However, as the data staging precedes training, each rank needs to hold all the data files it needs to access on the local storage. On the other hand, LBANN offers a built-in, in-memory data store in which sample data are transferred from the owner rank to the consumer rank as needed before every minibatch using non-blocking communication. This eliminates the redundant in-memory copies of data, hides the overhead in redistributing them and reduces the volume of the redistribution in case that each data file contains multiple samples.

\section{CONCLUSIONS}

In this paper we have presented novel improvements to the "Let a Thousand Flowers Bloom" (LTFB) torunament algorithm that allow it to train traditional and generative adversarial networks at scale. We have discussed how it is integrated into the LBANN open-source scalable deep learning framework, and demonstrated how it enables scalable training of a massive scientific data set. We have accelerated the training and discovery of novel neural network architectures for simulations of capsule implosions for inertial confinement fusion. Our tournament algorithm allows for the parallel training of a single neural network model that has learned on multiple silos of a partitioned data set. We show that as the number of parallel trainers increases, and thus the percentage of the data set show to each trainer decreases, LTFB enables strong scaling of the training time without loss of network quality. We are working with a very large scientific data set that has 10 million unique training samples with 120 million images and 150 million scalar values. To enable efficient parallel training we have developed an in-memory distributed data store that significantly improves the steady state per epoch training time. The aggregation of these technologies provides a scalable deep learning framework that is extremely well suited for parallel training on massive data sets. These capabilities provide the first step along the path of developing a cognitive simulation workflow.

\section{ACKNOWLEDGMENT}

This work was performed under the auspices of the U.S. Department of Energy by Lawrence Livermore National 
Laboratory under Contract DE-AC52-07NA27344 (LLNLCONF-677443). Funding partially provided by LDRD 18-SI002 .

\section{REFERENCES}

[1] B. K. Spears, J. Brase, P.-T. Bremer, B. Chen, J. Field, J. Gaffney, M. Kruse, S. Langer, K. Lewis, R. Nora, J. L. Peterson, J. Jayaraman Thiagarajan, B. Van Essen, and K. Humbird, "Deep learning: A guide for practitioners in the physical sciences," Physics of Plasmas, vol. 25, no. 8, p. 080901, 2018. [Online]. Available: https://doi.org/10.1063/1.5020791

[2] J. Gaffney, P. Springer, and G. Collins, "Thermodynamic modeling of uncertainties in NIF ICF implosions due to underlying microphysics models," in APS Meeting Abstracts, 2014.

[3] P. Springer, C. Cerjan, R. Betti, J. Caggiano, M. Edwards, J. Frenje, V. Y. Glebov, S. Glenzer, S. Glenn, N. Izumi et al., "Integrated thermodynamic model for ignition target performance," in EPJ Web of Conferences, vol. 59. EDP Sciences, 2013, p. 04001.

[4] B. Van Essen, H. Kim, R. Pearce, K. Boakye, and B. Chen, "LBANN: Livermore big artificial neural network HPC toolkit," in Proceedings of the Workshop on Machine Learning in HighPerformance Computing Environments (MLHPC), 2015. [Online]. Available: https://doi.org/10.1145/2834892.2834897

[5] T. Benson, N. Dryden, R. Forsyth, D. Hysom, S. A. Jacobs, N. Maruyama, D. McKinney, A. Moody, T. Moon, Y. Oyama, J.-S. Yeom, A. Yoo, and B. Van Essen, "Livermore Big Artificial Neural Network (LBANN) Toolkit,” 2019. [Online]. Available: https://github.com/LLNL/lbann

[6] T. Benson, T. Moon, J. Poulson, and B. Van Essen, "Hydrogen Distributed Linear Algebra Library," 2019. [Online]. Available: https://github.com/LLNL/Elemental

[7] N. Dryden, N. Maruyama, T. Moon, T. Benson, A. Yoo, M. Snir and B. Van Essen, "Aluminum: An asynchronous, GPU-aware communication library optimized for large-scale training of deep neural networks on HPC systems," in Proceedings of the Workshop on Machine Learning in HPC Environments (MLHPC), 2018.

[8] N. Dryden, N. Maruyama, T. Moon, T. Benson, A. Yoo, and B. Van Essen, "Aluminum GPU-aware communication library," 2019. [Online]. Available: https://github.com/LLNL/Aluminum

[9] R. Betti, A. Christopherson, B. Spears, R. Nora, A. Bose, J. Howard, K. Woo, M. Edwards, and J. Sanz, "Alpha heating and burning plasmas in inertial confinement fusion," Physical review letters, vol. 114, no. 25, p. 255003, 2015.

[10] M. M. Marinak, G. D. Kerbel, N. A. Gentile, O. Jones, D. Munro, S. Pollaine, T. R. Dittrich, and S. W. Haan, "Three-dimensional hydra simulations of national ignition facility targets," Physics of Plasmas, vol. 8, no. 5, pp. 2275-2280, May 2001. [Online]. Available: http://dx.doi.org/10.1063/1.1356740

[11] J. L. Peterson, K. D. Humbird, J. E. Field, S. T. Brandon, S. H. Langer, R. C. Nora, B. K. Spears, and P. T. Springer, "Zonal flow generation in inertial confinement fusion implosions," Physics of Plasmas, vol. 24, no. 3, p. 032702, 2017. [Online]. Available: https://doi.org/10.1063/1.4977912

[12] B. Kailkhura, J. J. Thiagarajan, C. Rastogi, P. K. Varshney, and P.-T. Bremer, "A spectral approach for the design of experiments: Design, analysis and algorithms," The Journal of Machine Learning Research, vol. 19, no. 1, pp. 1214-1259, 2018.

[13] I. Goodfellow, J. Pouget-Abadie, M. Mirza, B. Xu, D. Warde-Farley, S. Ozair, A. Courville, and Y. Bengio, "Generative adversarial nets," in Advances in Neural Information Processing Systems 27, Z. Ghahramani, M. Welling, C. Cortes, N. D. Lawrence, and K. Q. Weinberger, Eds. Curran Associates, Inc., 2014, pp. 2672-2680. [Online]. Available: http://papers.nips.cc/paper/5423-generative-adversarial-nets.pdf

[14] R. Anirudh, P.-T. Bremer, J. J. Thiagarajan, and USDOE National Nuclear Security Administration, "Cycle consistent surrogate for inertial confinement fusion," 2 2019. [Online]. Available: https: //www.osti.gov//servlets/purl/1510714

[15] S. A. Jacobs, N. Dryden, R. Pearce, and B. Van Essen, "Towards scalable parallel training of deep neural networks," in Proceedings of the Machine Learning on HPC Environments, ser. MLHPC'17. New York, NY, USA: ACM, 2017, pp. 5:1-5:9. [Online]. Available: http://doi.acm.org/10.1145/3146347.3146353
[16] J. Poulson, B. Marker, R. A. Van de Geijn, J. R. Hammond, and N. A. Romero, "Elemental: A new framework for distributed memory dense matrix computations," ACM Transactions on Mathematical Software, vol. 39, no. 2, p. 13, 2013.

[17] C. Harrison, B. Whitlock, and J. Ciurej, "Conduit:Simplified Data Exchange for HPC Simulations," 2019. [Online]. Available: https: //github.com/LLNL/conduit

[18] "TensorFlow: Data Input Pipeline," 2019. [Online]. Available: https: //www.tensorflow.org/guide/performance/datasets

[19] "PyTorch: DataLoader," 2019. [Online]. Available: https://pytorch.org/ docs/stable/data.html

[20] M. Jaderberg, V. Dalibard, S. Osindero, W. M. Czarnecki, J. Donahue, A. Razavi, O. Vinyals, T. Green, I. Dunning, K. Simonyan, C. Fernando, and K. Kavukcuoglu, "Population based training of neural networks," CoRR, vol. abs/1711.09846, 2017. [Online]. Available: http://arxiv.org/abs/1711.09846

[21] I. P. Durugkar, I. Gemp, and S. Mahadevan, "Generative multiadversarial networks," CoRR, vol. abs/1611.01673, 2016. [Online]. Available: http://arxiv.org/abs/1611.01673

[22] M. Abadi, A. Agarwal, P. Barham, E. Brevdo, Z. Chen, C. Citro, G. S. Corrado, A. Davis, J. Dean, M. Devin, S. Ghemawat, I. Goodfellow, A. Harp, G. Irving, M. Isard, Y. Jia, R. Jozefowicz, L. Kaiser, M. Kudlur, J. Levenberg, D. Mané, R. Monga, S. Moore, D. Murray, C. Olah, M. Schuster, J. Shlens, B. Steiner, I. Sutskever, K. Talwar, P. Tucker, V. Vanhoucke, V. Vasudevan, F. Viégas, O. Vinyals, P. Warden, M. Wattenberg, M. Wicke, Y. Yu, and X. Zheng, "TensorFlow: Large-scale machine learning on heterogeneous systems," 2015. [Online]. Available: https://www.tensorflow.org/

[23] N. Strom, "Scalable distributed DNN training using commodity GPU cloud computing," in INTERSPEECH, vol. 7, 2015, p. 10.

[24] Q. V. Le, R. Monga, M. Devin, K. Chen, G. S. Corrado, J. Dean, and A. Y. Ng, "Building high-level features using large scale unsupervised learning," in In International Conference on Machine Learning, 2012. 103, 2012.

[25] J. Dean, G. Corrado, R. Monga, K. Chen, M. Devin, M. Mao, A. Senior, P. Tucker, K. Yang, Q. V. Le et al., "Large scale distributed deep networks," in Advances in Neural Information Processing Systems, 2012.

[26] B. Recht, C. Re, S. Wright, and F. Niu, "Hogwild: A lock-free approach to parallelizing stochastic gradient descent," in Advances in Neural Information Processing Systems, 2011, pp. 693-701.

[27] A. Coates, B. Huval, T. Wang, D. Wu, B. Catanzaro, and N. Andrew, "Deep learning with COTS HPC systems," in International Conference on Machine Learning (ICML), 2013.

[28] F. N. Iandola, K. Ashraf, M. W. Moskewicz, and K. Keutzer, "FireCaffe: Near-linear acceleration of deep neural network training on compute clusters," arXiv preprint arXiv:1511.00175, 2015.

[29] P. Goyal, P. Dollár, R. Girshick, P. Noordhuis, L. Wesolowski, A. Kyrola, A. Tulloch, Y. Jia, and K. He, "Accurate, large minibatch SGD: training ImageNet in 1 hour," arXiv preprint arXiv:1706.02677, 2017.

[30] N. S. Keskar, D. Mudigere, J. Nocedal, M. Smelyanskiy, and P. T. P. Tang, "On large-batch training for deep learning: Generalization gap and sharp minima," in Proceedings of the Fifth International Conference on Learning Representations, 2017.

[31] M. Cho, U. Finkler, S. Kumar, D. Kung, V. Saxena, and D. Sreedhar, "PowerAI DDL," ArXiv e-prints, Aug. 2017.

[32] A. Karpatne, G. Atluri, J. H. Faghmous, M. Steinbach, A. Banerjee, A. R. Ganguly, S. Shekhar, N. F. Samatova, and V. Kumar, "Theoryguided data science: A new paradigm for scientific discovery," CoRR, vol. abs/1612.08544, 2016. [Online]. Available: http://arxiv.org/abs/ 1612.08544

[33] T. Kurth, S. Treichler, J. Romero, M. Mudigonda, N. Luehr, E. Phillips, A. Mahesh, M. Matheson, J. Deslippe, M. Fatica et al., "Exascale deep learning for climate analytics," in Proceedings of the International Conference for High Performance Computing, Networking, Storage, and Analysis, 2018.

[34] A. Mathuriya, D. Bard, P. Mendygral, L. Meadows, J. Arnemann, L. Shao, S. He, T. Karna, D. Moise, S. J. Pennycook et al., "CosmoFlow: Using deep learning to learn the universe at scale," in Proceedings of the International Conference for High Performance Computing, Networking, Storage, and Analysis, 2018. 